9-18-2019

\title{
Advancing Inclusion in the Geosciences: An Overview of the NSF- GOLD Program
}

Julie R. Posselt

Jason A. Chen

Grady Dixon

Jerlando F. L. Jackson

Robert Kirsch

See next page for additional authors

Follow this and additional works at: https://scholarworks.wm.edu/educationpubs

Part of the Scholarship of Teaching and Learning Commons, and the Science and Mathematics Education Commons 


\section{Authors}

Julie R. Posselt, Jason A. Chen, Grady Dixon, Jerlando F. L. Jackson, Robert Kirsch, Anne-Marie Nuñez, and Brian J. Teppen 


\title{
Authors
}

Dr. Julie R. Posselt, Associate Professor, University of Southern California, Rossier School of Education, 3470 Trousdale Parkway, 602g WPH, Los Angeles, CA 90089, Phone: 213-7406865; Email: posselt@usc.edu

Dr. Jason Chen, Gerdelman Family Term Distinguished Associate Professor of Education, College of William \& Mary, School of Education, 301 Monticello Ave., Williamsburg, VA 23185. Phone: 757-221-6201. Email: jachen@email.wm.edu

Dr. Grady Dixon, Professor and Chair, Department of Geosciences, Fort Hays State University, 600 Park St, Hays, KS 67601, Phone: 785-628-4536; Email: pgdixon@fhsu.edu

Dr. Jerlando F. L. Jackson, Vilas Distinguished Professor of Higher Education, Department Chair of Educational Leadership and Policy Analysis, School of Education, University of Wisconsin-Madison; 575 Educational Sciences; 1025 W. Johnson Street; Madison, WI 53706. Email: jjackson@education.wisc.edu

Dr. Robert Kirsch, Assistant Professor, Arizona State University, College of Integrative Sciences and Arts, 651 E University Dr., Tempe, AZ 85287, Phone: 480-727-3262 Email: rekirsch@asu.edu

Dr. Anne-Marie Nuñez, Professor, The Ohio State University, College of Education and Human Ecology, 29 W. Woodruff Avenue, Columbus, OH 43202. Email: nunez.80@osu.edu Dr. Brian J. Teppen, Professor, Michigan State University, College of Agriculture and Natural Resources, 1066 Bogue Street, Room 283, East Lansing, MI 48824, Phone: 517-528-3370; Email: teppen@msu.edu

\begin{abstract}
Here we report on five pilot projects working to develop effective professional development aimed at improving diversity, equity, and/or inclusion within the geosciences. All five projects were funded by the NSF GEO Opportunities for Leadership in Diversity (GOLD) program, which was designed to bring together geoscientists and social scientists to create innovative pilot programs for preparing and empowering geoscientists as change agents for increasing diversity. Each project has different objectives and applies different combinations of methods, but focus on professional development, bystander intervention training, and the formation of new networks in the pursuit of systemic, institutional change. This paper describes the origins, aims, and activities of these projects, and reflects on lessons learned to date. These projects are still ongoing, but in their first two years have received more interest than anticipated and more demand than can be fulfilled, suggesting an unserved need in the field. We have also found that teams with varied backgrounds, experiences, and expertise are vital to overcoming common struggles in facing inequalities. Coaching from experts in diversity, equity, and inclusion keeps the teams motivated, particularly when many team members are accustomed to typical scientific research. Finally,
\end{abstract}


40 institutional change requires time to catalyze, develop, and institutionalize, highlighting the

41 importance of sustained effort over years.

42

Keywords: Geosciences, Equity, Diversity, Inclusion, Leadership, Ideas Lab

47 The share of women earning geoscience degrees has reached 40 percent (Wilson, 2018),

48 representing a significant increase over the past four decades (Bernard \& Cooperdock, 2018), but

49 the discipline still lags behind STEM fields as a whole (NSF, 2017). Over the past 40 years,

50 about $85 \%$ of US Citizen $\mathrm{PhD}$ recipients in the geosciences have come from White, non-

51 Hispanic backgrounds, whereas 7\% have come from racially/ethnically underrepresented groups

52 (i.e., African American, Hispanic/Latinx, and Native American) (Bernard \& Cooperdock, 2018).

53 The remainder are mostly Asian-Americans, and a smaller number who do not identify with a

54 single race/ethnicity. The share of underrepresented students earning bachelor's degrees in the

55 geosciences in 2017 was 8\% (Wilson, 2018). Over the past 40 years, the proportion of

56 underrepresented groups earning degrees in the discipline has not changed (Bernard \&

57 Cooperdock, 2018).

58 Recognizing the need for systemic change to disrupt these trends and encourage

59 diversity, equity, and inclusion, NSF-GOLD was established. The name "GOLD” (GEO

60 Opportunities for Leadership in Diversity) is derived from the Directorate for Geosciences

61 (GEO) and the desire to identify and enhance discipline-specific leaders who can make positive

62 changes in the area of diversity. More specifically, the purpose of GOLD is to prepare and

63 empower scientists to become agents of change for increasing diversity. GOLD Program leaders

64 at NSF employed a novel method, called an "Ideas Lab", for achieving these goals.

\section{Origins in the Ideas Lab Format}


The NSF Ideas Lab is a method to encourage novel questions and ideas from a diverse group of participants. Participants meet for 5 full days of intense activities designed to challenge,

68 frustrate, stir emotions, and force collaboration. There is little or no agenda shared with

69 participants in advance, and the result is a series of research or other project proposals. The

70 concept was originally created in the U.K. in 2003, where they are called "Sandpits," and NSF

71 has been using Ideas Labs since 2009.

For the GOLD Ideas Lab, NSF invited a diverse group of 30 participants - geoscientists,

73 social scientists, and other practitioners working in STEM. Led by Knowinnovation, a company

74 that commonly facilitates Ideas Labs and Sandpits (https://knowinnovation.com), the end goal

75 for all this creative energy was for these 30 individuals to design and develop collaborative

76 professional development programs that could be field tested with small groups of geoscientists,

77 with the goal of using these pilot projects as a proof-of-concept to scale up. NSF, in consultation

78 with Knowinnovation, selected geoscientists as well as those with expertise in behavioral

79 change, social psychology, leadership development, and other related areas. NSF also brought in

80 a director and five "mentors" who had histories of working in the areas of diversity, equity, and

81 inclusion in science. Although the mentors participated fully in the Ideas Lab, they were not

82 eligible for funding because they were also acting as impartial reviewers of the participants

83 working toward proposals.

84 Every day of the Ideas Lab was densely scheduled with breaks for little more than three

85 meals from 8:00 a.m. until 8:00 p.m., and most participants continuing to work together until

86 around midnight each day. This process resulted in several great ideas. Many participants had at

87 least some role in multiple ideas, and not all great ideas were fully developed. Nevertheless, by

88 the end of the week, participants pitched the most feasible and most popular six proposals were 
89 to a panel of NSF program officers. Based on these presentations, NSF invited the most

90 promising projects to submit full proposals to NSF. Five proposals were eventually funded.

\section{GOLD Projects}

92 The author team of this commentary includes at least one PI from each of the projects that are

93 funded by NSF GOLD. The projects each seek to build leadership capacity of individual

94 scientists and the organizations in which they work. Collectively, the projects capture the

95 multiple contexts in which geoscience education occurs, and aim to:

96 Cultivate an ethical model of community-based geoscience research,

97 Create more equitable cultures of geoscience fieldwork,

98 Leverage the influence and wisdom of senior scholars toward inclusion,

99 Empower faculty to recognize and respond to prejudice in workplaces,

100 Change departmental culture by supporting small groups of change agents

101 In what was an initially discomforting aspect of the initiative, NSF representatives pushed the

102 PIs to orient projects toward engaging with parties other than students — namely, toward people

103 with power to make decisions that affect student participation. This conceptual orientation is

104 consistent with evidence that organizational change can happen by changing the mindsets and

105 practices of gatekeepers and other leaders (Bensimon, 2005; Kezar, 2012; Posselt, 2016).

106 In this respect, the inclusion of both geoscientists (about 75\%) and social scientists (about

$10725 \%$ ) on project teams has been a defining feature of GOLD. It enables a crucial strategy across

108 these diverse projects: equipping geoscientists, who are not trained in social science, with current

109 theories for: (1) discussing diversity, equity, and inclusion; (2) implementing effective

110 educational practices; and (3) effecting changes toward these goals. Well-intentioned

111 geoscientists may know, for example, the importance of saying something when they see 
112 harassment or assault, but rarely do they know quite how to handle such situations when they

113 arise. Training in bystander intervention in three of the five GOLD projects has begun to

114 empower scientists with knowledge and skills to make their work more inclusive.

115 Summaries of Projects, Research, \& Theories

116 In addition to their shared origins in the Ideas Lab process and multidisciplinary

117 leadership teams, the initial GOLD projects have conceptual and programmatic similarities,

118 which are summarized in Table 1. All five include professional development opportunities for

119 participants, and are oriented toward the broad aim of changing institutional culture. Three of the

120 five create new social or professional networks, all five explicitly address equity, diversity, and

121 inclusion as systemic issues, and three offer bystander intervention training as part of

122 professional development. 


\section{Table 1. Features of NSF-GOLD Pilot Projects}

\begin{tabular}{llllll}
\hline Project & $\begin{array}{l}\text { Professional } \\
\text { Development } \\
\text { Included }\end{array}$ & $\begin{array}{l}\text { Bystander } \\
\text { Intervention } \\
\text { Training }\end{array}$ & $\begin{array}{l}\text { Explicitly } \\
\text { Addresses } \\
\text { Systemic } \\
\text { Issues }\end{array}$ & $\begin{array}{l}\text { Forms New } \\
\text { Social or } \\
\text { Professional } \\
\text { Networks }\end{array}$ & $\begin{array}{l}\text { Changes } \\
\text { Institutional } \\
\text { Culture }\end{array}$ \\
\hline ASPIRE & $\mathrm{Y}$ & $\mathrm{Y}$ & $\mathrm{Y}$ & $\mathrm{N}$ & $\mathrm{Y}$
\end{tabular}

Novelty: Community-engaged science broadens vision of what and whom science is for; Social science research design contributes to reflection and growth by mobile working group leaders
FIELD
$\mathrm{Y}$
Y
$\mathrm{Y}$
Y
Y

Novelty: Focus on field work; Participants were leaders of field experiences at a variety of institutions, representing a full range of career stages.
GeoDES
$\mathrm{Y}$
Y $\quad \mathrm{Y}$
$\mathrm{N} \quad \mathrm{Y}$

Novelty: Mixed-reality simulations to help geoscience faculty identify prejudices and prejudicial structures, and then take action to redress those issues.

$\begin{array}{llllll}\text { Hearts of Gold } & \text { Y } & \text { N } & \text { Y } & \text { Y } & \text { Y }\end{array}$

Novelty: Multiple cohorts of senior scientists learning how to use their influence to spread allophilia (i.e., love of those who are different) in support of healthier cultures for diverse students and colleagues

$\begin{array}{llllll}\text { Sparks for Change } & \text { Y } & \text { N } & \text { Y } & \text { Y } & \text { Y }\end{array}$

Novelty: Mentoring triads of early-career faculty from an underrepresented group, senior faculty, and external expert on broadening participation

\section{ASPIRE (Active Societal Participation in Research and Education)}

A common paradigm for science is discovery emanating from curiosity about the natural world, carried out by testing discipline-specific theory. Disenfranchised communities, however, may see problems emanating from environmental injustices as more relevant than theory-

130 generating research as relevant; therefore, a growing number of scientists are moving out of the

131 ivory tower, and directly into communities to conduct geoscience research that addresses

132 community-identified problems that are both social and scientific. ASPIRE pilots a model of

133 place- and community-based geoscience research distinguished by scientists and community

134 members, facilitated by an individual who has a foot in both worlds. Together, they collaborate

135 in an ethical exchange of knowledge, values, and cultural perspectives about one specific 
136 ecological, environmental, or geoscience challenge. ASPIRE supports six mobile working

137 groups of scientists and community members addressing local challenges using the tools of

138 geoscience. Presently, three groups are supported for research in South Dakota, Hawaii, and Los

139 Angeles, with three more to start up in 2019. One project, led by Dr. Andres Aguilar of

140 California State University- Los Angeles, engages community members who live, work, and

141 play along the Los Angeles River to measure the chemical composition of river water and its

142 safety for various purposes. Their measurements are informing Aguilar's scholarship and

143 clarifying appropriate uses of the river water. Another study brings together a family-owned

144 watercress farm just outside of Honolulu with researchers from the University of Hawaii-Manoa

145 to understand how urbanization and changing water policy are affecting the water quality on

146 their farm and, by extension, the crops and the indigenous farm's sustainability.

147 How projects and others, in practice, resemble the idealized model of ethical place-based

148 research is one topic of research underway. Another research aim is advancing knowledge of

149 what leadership of place-based, community-based science entails. ASPIRE uses cultural

150 sociological theories about social and symbolic boundaries to understand how working group

151 leaders operate as boundary spanners, linking the cultures and approaches to knowledge

152 production among communities and geoscientists. Mobile working groups of principal

153 investigators are participating in a narrative inquiry over the course of their project-- including

154 extended pre-post interviews as well as writing monthly logs on issues of common concern. The

155 research model ASPIRE pilots strives to integrate both scientists' sensibilities and those of

156 communities who have been excluded or opted out of the geosciences.

157 FIELD (Fieldwork Inspiring Expanded Leadership for Diversity) 
Fieldwork is a central activity for geoscience learning, and has been characterized as "a

159 key benchmark in the transition from student to scientist and from novice to expert” (Feig, 2010,

160 p. 249). However, cultural dynamics like financial cost, anxiety about outdoor experiences,

161 attitudes of ableism, and threat of sexual assault also prevent some people from entering or

162 continuing in the discipline. The association of geosciences with outdoor activity can hinder the

163 engagement of some populations, including those from black, Latinx, and indigenous

164 backgrounds (e.g., O’Connell \& Holmes, 2011; Stokes, Levine, \& Flessa, 2015). For these

165 populations, the wilderness can be associated with human histories of natural resource extraction,

166 military expansion, labor exploitation, colonization by settlers, and dispossession of land, each of

167 which has been facilitated in some part by disciplinary practices of geosciences (e.g., Whyte,

168 2017; Yusoff, 2018). For example, in the U.S., slavery and Jim Crow laws made the rural

169 outdoors dangerous for African Americans, especially in the South (e.g., Edmondson, 2006;

170 Finney, 2014; Hackmann, 2015); concern about safety in rural areas remains a legacy of this

171 history for some African American college students today. Globally, the mining of natural

172 resources, such as gold, coal, and tin, has been associated with exploitative labor with Asian,

173 Black, and Indigenous peoples (Whyte, 2017; Yusoff, 2018). FIELD aims to raise awareness of

174 historical, engrained barriers in geoscience field experiences and to make field activities more

175 inclusive by equipping leaders with perspective, skills, and solidarity.

176 The initial phase of the project involved ethnographic research by social scientists (i.e.,

177 authors Nuñez and Posselt) in an undergraduate field camp and graduate-level field course.

178 Findings highlighted how typical classroom or lab boundaries break down in the field, with

179 positive and negative consequences for inclusivity. Fieldwork demands of generating hypotheses

180 about the formation of landscapes inspired cognitive, social, and physical disequilibria that can 
181 inspire unique learning (also see Feig, 2010; Mogk \& Goodwin, 2012), but also requires

182 instructors to attend all students' wellbeing and engagement. Working long hours in high

183 temperatures, for example, put students in one of the courses at risk for heat stroke.

184 Next, the FIELD project convened a three-day leadership institute for faculty and field-

185 camp geoscientists. Drawing on the research of the project's first phase, the FIELD Institute at

186 Colorado State University's Mountain Campus offered training in practical skills like bystander

187 intervention, managing cross-cultural relationships, and more. It also facilitated opportunities for

188 collaborative development of new approaches to fieldwork. The final phase will consist of

189 evaluation, assessment, and construction of a professional leadership model based on results of

190 the FIELD Institute. FIELD Institute participants will work together to interrupt and advance

191 new alternatives to these dynamics in their own sites, and the leadership team intends to generate

192 models for inclusive fieldwork that can be adapted across all educational levels.

\section{GeoDES (Geoscience Diversity Experiential Simulations)}

194 GeoDES aims to provide professional development for a cohort of 30 geoscientists to

195 develop their (1) knowledge of social justice issues in geosciences; (2) bystander intervention

196 skills; and (3) leadership skills for targeting exclusionary gatekeeping decisions. Critical

197 elements of the approach included an intensive three-day workshop and three mixed-reality

198 simulations, which combine human conversational intuition with artificial intelligence. The

199 "human-in-the-loop" architecture used in GeoDES simulations provide highly authentic and

200 realistic scenarios that allow project participants to learn and practice specific skills and

201 strategies that they learn throughout the project. For example, one simulation provided

202 participants with the opportunity to learn and practice how to recognize biases that arise in

203 faculty search committees and then to advocate for promising candidates who do not possess 
204 such traditionally valued experiences as graduating from a prestigious elite university, publishing

205 in a journal that the committee members recognize, and having a well-known mentor. On-going

206 professional development also included monthly virtual meetings in which participants discussed

207 applying their knowledge and skills to their home institutions.

208 To date, the GeoDES team has collected longitudinal survey data at four time points

209 spanning one year of participation. We (i.e., authors Chen, Jackson, and Teppen and the

210 GeoDES leadership team) have also collected audio and video data of our participants engaging

211 in the mixed-reality simulations. Although we are in the beginning stages of data analysis, we

212 have some preliminary findings from the quantitative data. Using a Bayesian analytical

213 approach, we found that participants' $(n=29)$ beliefs about their individual capabilities (i.e., self-

214 efficacy) and beliefs about their department's collective capabilities (i.e., collective efficacy) to

215 confront prejudices and prejudicial structures grew sharply from the start of the project

$216[$ Mean $($ self-efficacy $)=3.45 ;$ Mean $($ collective efficacy $)=3.14]$ to three months after starting

$217[$ Mean $($ self-efficacy $)=4.38 ;$ Mean(collective efficacy $)=3.96]$. Although self-efficacy had declined

218 to a mean of 3.78 and collective efficacy to 3.28 one year later, there was still overall growth

219 from start to end. To put more fine-grained details to this overall landscape of self-reported

220 changes in beliefs, we plan to use the video data of participants in the three simulations to

221 explore the variety of ways that participants approached the situations presented to them in the

222 simulations and the degree to which they challenged prevailing norms tactfully.

223 Ideally, GeoDES participants develop multiple habits for leading organizational-level

224 change by: a) intervening whenever exclusionary behavior is witnessed, b) strategically

225 advocating for marginalized voices when participating in key gatekeeping decisions such as

226 hiring, and c) changing institutional policies such as annual merit review processes to reward 
227 people who engage in diversity, equity, and inclusion efforts. Participants learn a social closure

228 model (Murphy, 1988) that helps articulate the processes by which exclusion occurs. Further,

229 GeoDES participants engaged in mixed-reality simulations in which they practiced all three of

230 these forms of leading change. Research can inform the scaling up of this type of innovation to

231 larger groups, thereby generating broader impacts on other fields.

232 Hearts of GOLD

233 Hearts of GOLD aims to develop and test professional development training for

234 established scientific leaders in the geosciences - the GOLD Institute - to give them the

235 content knowledge, tools, and skills needed to become champions of change for diversity. It is

236 hosted in Colorado Springs and facilitated by Drs. Dena Samuels and Stephany Rose of the

237 Knapsack Institute at the University of Colorado- Colorado Springs.

238 Its task is to move attitudes among opinion leaders in the geosciences away from negative

239 intergroup attitudes to positive ones. Pittinsky (2005) describes those positive intergroup

240 attitudes as "allophilia," borrowed from the Greek for "love of the other." We need to foster

241 those positive attitudes among opinion leaders, because they can promote behavior change

242 among those around them (Valente and Pumpuang, 2007). Its method is inspired by Wenger's

243 Communities of Practice (Wenger, 2000) where leaders set the values for the community but

244 often have little access to other communities to bring in new ideas. The GOLD Institute provides

245 this access by hosting two cohorts that receive a two-day, transformative, intensive workshop

246 targeted at existing scientific leaders to develop them as leaders in diversity. These leaders will

247 promote scalable change by taking action to promote allophilia within their home institutions and

248 within their roles as members of professional societies. Furthermore, participants will form a 
249 national network of geoscientists who support each other in extending allophilia throughout the 250 geosciences.

251 Hearts of GOLD employs the most conservative strategy of the GOLD projects, but has a

252 multi-cohort model that will help promote grassroots growth over time. Accordingly, five

253 participants from the first cohort joined those in the second to help bridge the two groups.

\section{Sparks for Change}

The Sparks for Change team uses a model of small group dynamics so early-career,

256 underrepresented minority faculty can become effective leaders in changing department culture

257 concerning diversity, equity, and inclusion in the geosciences. These groups consist of an early-

258 career, underrepresented minority faculty member, and senior faculty member from the same

259 unit, as well as an expert on broadening participation who is external to that unit. Each member

260 of the small group emphasizes a specific leadership style relevant to their position in the

261 department. The project held a three-day workshop in 2017 to highlight these leadership styles,

262 build social bonds within and among small groups for post-workshop support, and develop

263 action plans that utilize leadership insights to change department culture toward diversity, equity,

264 and inclusion.

265 Research begins with identifying an institutional inertia in geoscience departments.

266 Although many departments are in principle open to diversity, equity, and inclusion, there may

267 nevertheless be benefits to maintaining the status quo. Overcoming the diffuse benefits of the

268 status quo requires group of active, empowered change agents. The project hypothesizes that

269 small groups of committed change agents will change the department culture with a concrete

270 action plan in order to overcome that inertia (Bergstrom, 2010). This is an emergent approach to

271 changing department culture specific to the institution where change agents are located and does 
272 not focus on top-down directives; rather, leadership development unlocks the skills and

273 capacities that small group members already possess and asks them to leverage those skills to

274 effect change.

275 A promising possibility from the Sparks model is its potential for proliferation. It is 276 lightweight and scalable, and shows how accomplishing small goals might be scaled up to

277 broader contexts (McGinnis \& Ostrom, 2007). Another aspect of this research is the role of the 278 outside expert, who may keep the group focused on breaking inertia and enabling broader 279 change.

\section{Reflections and Recommendations}

Though data collection and the required external project evaluation are still underway in

282 a majority of the projects, we have learned that project similarities are more than a function of

283 the common funding stream and our shared focus on building capacity for diversity leadership.

284 Several noteworthy patterns have emerged. First, although our small pilot projects were funded

285 to accept only 30 participants, our projects received far more requests to participate than we were

286 able to accept. In fact, some participants even volunteered to pay their own way to attend

287 institutes and trainings associated with the projects — clear indications of hunger within the

288 geosciences to learn how to facilitate change and enact values of diversity, equity, and inclusion.

289 Upon attendance, participants have eagerly engaged with the opportunities for professional

290 development that these projects offer, which include network formation, self-reflection, dialogue,

291 and bystander intervention training. Simply learning to notice and label bias can lead to more

292 inclusive attitudes and (perhaps) actions (Forscher et al., 2017). Equipping scientists with social

293 knowledge and skills appears to be an important, underrecognized lever for change. 
As the projects mature, we will also be attending to barriers to sustainable change within scientific and educational institutions, such as those related to the existing academic incentive structures. For example, in FIELD, leaders may be committed to more inclusive practices, but

297 these may increase financial costs in a time that funding for fieldwork is already threatened or 298 declining. We will have more to report on barriers by the end of the pilot phase, but anticipate 299 now that clarity about these barriers will only reinforce GOLD's focus on systemic change.

300 For other geoscience educators engaged in diversity, equity, and inclusion efforts, we can

301 make a few early recommendations: First, geoscience education is inherently interdisciplinary,

302 and the problems of exclusion and lack of diversity are inherently social. We thus endorse

303 GOLD's vision of leadership teams representing diverse social identities and both social and

304 hard sciences, who bring distinctive knowledges and ways of knowing to the work. Social

305 scientists have brought in new research tools and practices, enhanced the awareness and

306 intentionality of research design, and allowed for more rigorous evaluation. Geoscientists on

307 each project ensure alignment of efforts with geoscience cultures and translate social science

308 concepts to participants. PIs, participants, and coaches alike are becoming geo-social-science

309 boundary spanners. For example, all of the authors of this manuscript continue working closely

310 across disciplines, supporting and learning from each other.

311 Second, diversity does not come without its challenges--differing disciplinary cultural

312 norms and social identities may yield misunderstandings within even high-functioning teams.

313 We therefore recommend leadership teams include external advisors or coaches. For example,

314 during the Ideas Lab, even though participants were a diverse group with diverse expertise, NSF

315 was intentional about hiring an external group to facilitate the creative idea-generation while

316 keeping participants within the boundaries of what the program would realistically fund. 
317 Furthermore, as the projects started and matured, NSF funded two coaches, Diana Kardia and

318 Kelly Mack, to help manage potential conflicts among and within GOLD groups so that our

319 diversity could be transformed into products such as a podcast series that explores the skills and

320 experiences required to lead efforts to broaden participation. Please see Kardia Group, LLC

321 (2018)

322 Finally, work like this takes time. Therefore, we recommend project development on the

323 time scale of at least three to five years, particularly if leaders have not previously worked

324 together. In our own experience, with more participants, a longer timeline, more substantial

325 funding, and more cross-fertilization among the projects, our potential for impact would no

326 doubt be greater.

Acknowledgements

328 The authors on this manuscript would each like to thank their fellow principal investigators on

329 their respective GOLD projects, as well as the National Science Foundation for financial support

330 of these efforts.

\section{References}

Bensimon, E. M. (2005). Closing the achievement gap in higher education: An organizational learning perspective. New directions for higher education, 2005(131), 99-111.

Bergstrom, T. C. (2010). The uncommon insight of Elinor Ostrom. The Scandinavian Journal of Economics, 112(2), 245-261.

Bernard, R.E., \& Cooperdock, E. H. G. (2018). No progress on diversity in 40 years. Nature, 11, 292-295. https://doi.org/10.1038/s41561-018-0116-6.

Edmondson, D. (2006). Black and brown faces in America's wild places. Cambridge, MN: Adventure Publications.

Feig, A. D. (2010). Technology, accuracy, and scientific thought in field camp: An ethnographic study. Journal of Geoscience Education, 58(4), 241-251.

Finney, C. (2014). Black Faces, White Spaces: Reimagining the Relationship of African Americans to the Great Outdoors. Chapel Hill, NC: University of North Carolina Press. 
Forscher, Patrick S., Chelsea Mitamura, Emily L. Dix, William T.L. Cox, and Patricia G. Devine. 2017. Breaking the prejudice habit: Mechanisms, timecourse, and longevity. $J$ Experimental Social Psychology 72,133-146.

Hackman, R. (2015, August 4). Swimming while Black: The legacy of segregated swimming pools lives on. The Guardian. Retrieved from: https://www.theguardian.com/world/2015/aug/04/black-children-swimming-drowningssegregation

Kardia Group LLC (2018, October 18). Leadership for broadening participation podcast series. Retrieved from https://kardiagroup.com/leadership-for-broadening-participation-podcastseries/

Kezar, A. J. (2012). Shared leadership for creating campus cultures that support students of color. In Creating Campus Cultures (pp. 160-177). Routledge.

McGinnis, M., \& Ostrom, E. (2007). Will lessons from small-scale social dilemmas scale up? In A. Biel, D. Eek, T. Gärling, \& M. Gustafson (Eds.), New issues and paradigms in research on social dilemmas (pp. 189-211). Springer Science \& Business Media.

Mogk, D., Goodwin, C., 2012. Learning in the field: Synthesis of research on thinking and learning in the geosciences. In Kastens, K.A., \& Manduca, C.A. (eds.) Earth and Mind II: A Synthesis of Research on Thinking and Learning in the Geosciences. Geological Society of America Special Papers. 486:131-164.

Murphy, Raymond. 1988. Social closure: The theory of monopolization and exclusion. Clarendon Press, Oxford. 276 p.

National Science Foundation (NSF) (2017). Women, Minorities, and Persons with Disabilities in Science and Engineering: 2017. Special Report NSF 17-310. Arlington, VA: National Center for Science and Engineering Statistics. Retrieved Apr. 24, 2018, from www.nsf.gov/statistics/wmpd/

O’Connell, S., \& Holmes, M. A. (2011). Obstacles to the recruitment of minorities into the geosciences: A call to action. GSA Today, 21(6), 52-54.

Pittinsky, T. L. (2005). Allophilia and Intergroup Leadership. Kennedy School of Government Working Paper, No. RWP05-038.

Posselt, J. R. (2016). Inside graduate admissions: Merit, diversity, and faculty gatekeeping. Cambridge, MA: Harvard University Press.

Stokes, P.J., Levine, R., \& Flessa, K. W. (2015). Choosing the geoscience major: Important factors, race/ethnicity, and gender. Journal of Geoscience Education, 63(3), 29-40.

Valente, T. W., \& Pumpuang, P. (2007). Identifying opinion leaders to promote behavior change. Health Education \& Behavior, 34(6), 881-896.

Whyte, K. (2017). Indigenous climate change studies: Indigenizing futures, decolonizing the Anthropocene. English Language Notes, 55 (1-2), 153-162.

Wilson, C. (2018). Status of recent geosciences graduates 2017. Washington, DC: American Geosciences Institute. Retrieved from: https://www.americangeosciences.org/sites/default/files/ExitSurvey_2017_Online_04101 8.pdf 
387 Wenger, E. (2000). Communities of practice and social learning systems. Organization, 7(2), $388 \quad 225-246$.

389 Yusoff, K. (2018). A billion black Anthropocenes or none. Minneapolis, MN: University of $390 \quad$ Minnesota Press

391

392 
395 [1] We use women/men for NSF gender identity statistics, reserving the language of female/male 396 for instances where biological distinctions are relevant to the discussion at hand.

[2] For details and history of the Ideas Lab format within NSF, please see https://nsf.gov/discoveries/disc_summ.jsp?cntn_id=136669. For details of the original

400 solicitation by NSF-GEO, please see https://www.nsf.gov/pubs/2016/nsf16516/nsf16516.htm

401

402 